\title{
Artificial Whiskers Suitable for Array Implementation: Accounting for Lateral Slip and Surface Friction
}

\author{
Joseph H. Solomon and Mitra J. Z. Hartmann
}

\begin{abstract}
The exquisite tactile sensing ability of biological whiskers has recently led to increasing interest in constructing robotic versions with similar capabilities. Tactile extraction of three-dimensional (3-D) object shape poses several unique challenges that have only begun to be addressed. The present study develops a method for estimating the contact location of a robotic whisker rotating against an object based on small changes in moment at the whisker base. Importantly, the method accounts for lateral slip as well as surface friction, making it particularly well suited for implementation on an array of robotic whiskers. Array implementation would permit simultaneous extraction of multiple contact points and enable highly parallel, efficient 3-D object feature extraction. A simple, scalable array design is suggested to fulfill this approach.
\end{abstract}

Index Terms-Biomimetic whisker, feature extraction, flexible beam, tactile sensing.

\section{INTRODUCTION}

$\mathbf{F}$ ACIAL and body whiskers serve as a remarkably sensitive source of tactile information for many species of mammals [1]-[16]. For example, harbor seals can use their facial whiskers to track hydrodynamic trails, an ability thought to aid longdistance prey tracking [6]. Behavioral experiments on rats have shown that individual whiskers provide the fine-grained distance discrimination sensitivity needed to sense aperture width [16]. Increased study of the biological function of whiskers has been paralleled by an increasing interest in constructing their robotic counterparts [17]-[23]. Robotic whisker arrays could be used in a wide variety of applications, ranging from obstacle-avoidance and navigation tasks that require only coarse sensing capabilities to sensing tasks that push the limits of tactile discrimination, and hence require engineers to construct systems that rival animals' exquisite tactile sensitivity.

The simplest types of artificial whiskers are used as binary contact detection sensors [24]-[26], which can be used in tasks such as obstacle avoidance [27]. These types of whiskers have been used successfully on several toys (e.g., the BioBug from WowWee toys). Other researchers have explored the use of arti-

Manuscript received November 1, 2007; revised February 28, 2008. First published September 23, 2008; current version published October 31, 2008. This paper was recommended for publication by Associate Editor K. Iagnemma and Editor F. Park upon evaluation of the reviewers' comments. This work was supported in part by the Bio-Inspired Technologies and Systems/CISM at the National Aeronautics and Space Administration's (NASA) Jet Propulsion Laboratory and by the National Science Foundation (NSF) under Grants IOB-0446391 and IIS-0613568.

The authors are with the Department of Biomedical Engineering and the Department of Mechanical Engineering, Northwestern University, Evanston, IL 60208 USA (e-mail: m-hartmann@ northwestern.edu).

Digital Object Identifier 10.1109/TRO.2008.2002562 ficial whiskers for wall following [26], for terrain mapping [28], to characterize surface texture [17], [18], [23], [28], [29] and surface defects [30], to sense fluid flow profiles [22], and to investigate questions in neuroscience [22], [23], [29]. Most recently, a whisker sensor was designed for precise three-dimensional (3-D) measurement of heart position in robot-assisted beating heart surgery [31].

An important feature of biological whiskers is their ability to extract 3-D features, either of solid objects or of fluid flows. Several studies have investigated how this capability might be replicated in an artificial whisker array. Tsujimura and Yabuta [32] showed that a stiff, insensitive probe attached to a six-axis force/torque sensor can be used to measure probe-object contact positions along 3-D objects. Russell [33] attached a curved, binary whisker to a Puma robot to sweep along and estimate object contours. Wilson and Chen [34] used a pneumatic bellow tube actuation system to sweep a flexible spring steel wire whisker instrumented with strain gages at the base along objects and estimate their profiles. Ueno et al. [35] measured the vibration frequencies of a flexible beam with a payload at the tip to estimate contact point position with an object. Kaneko et al. [19] actuate a flexible beam while measuring bending moment at the base to determine contact distance based on the rotational compliance. Russell and Wijaya [36] develop a geometric approach to feature extraction using an array of eight rigid rods with angle sensors. Scholz and Rahn [21] actuate a Nitinol whisker attached to a mini load cell that measures both force and torque to achieve continuous estimation of 2-D whisker shape, and thus, the surface profile as it sweeps along objects. Clements and Rahn [37] used a similar setup along with a two-axis robot to gather discrete contact points while sweeping a whisker along unknown objects. Kim and Möller [20] explore the advantages of using multiple whiskers with the ability to measure both horizontal and vertical deflections for shape recognition. Table I summarizes several of the whisker sensor technologies that have been successfully implemented and the way in which they were used. In all cases, the actual sensing device resides at or near the base of an insensitive beam (the "whisker"), and a motor is typically used for actuation.

Despite the significant number of studies suggesting the possibility of 3-D feature extraction with whiskers, there are two interrelated problems that have as yet prevented artificial whiskers from being used in large, highly parallel, actuated arrays to sense object features. The first problem is lateral slip, in which the whisker slides out of its plane of rotation, thus preventing an accurate measurement of object distance. The second problem, 
TABLE I

WHISKER SENSOR TECHNOLOGIES

\begin{tabular}{|l|l|}
\hline \hline \multicolumn{1}{|c|}{ Sensor } & \multicolumn{1}{|c|}{ Description } \\
\hline Binary & $\begin{array}{l}\text { An electrode housing at the base detects the presence } \\
\text { (ON) or lack (OFF) of contact with the whisker. Binary } \\
\text { whiskers are usually used for contact detection [24- } \\
\text { 26], but have also been used to sense contours [33]. }\end{array}$ \\
\hline $\begin{array}{l}\text { Capacitor } \\
\text { microphone }\end{array}$ & $\begin{array}{l}\text { Forces and moments transmitted to the whisker base } \\
\text { lead to deformation of a microphone membrane, which } \\
\text { has been used to measure surface texture [17, 29] and } \\
\text { avoid obstacles [27]. }\end{array}$ \\
\hline Strain gage & $\begin{array}{l}\text { Strain gages are mounted either on a block or on thin } \\
\text { plates to measure bending moment at the base of the } \\
\text { whisker. They have been used to measure both contact } \\
\text { points [22, 28,31,34] and texture [23, 28]. }\end{array}$ \\
\hline $\begin{array}{l}\text { Six-axis } \\
\text { force/torque } \\
\text { load cell }\end{array}$ & $\begin{array}{l}\text { Load cells can measure all three components of both } \\
\text { force and moment and have been used to estimate } \\
\text { contact points [32, 37] and continuous object profiles } \\
\text { [21]. They provide the most information, but tend to } \\
\text { be bulky and expensive. }\end{array}$ \\
\hline Piezoelectric & $\begin{array}{l}\text { Piezoelectric sensors produce signals directly } \\
\text { proportional to rate of change of moment at the } \\
\text { whisker base [20]. }\end{array}$ \\
\hline Hall effect & $\begin{array}{l}\text { Hall effect sensors measure magnetic flux caused by } \\
\text { deflection of the whisker near the base (i.e. moment), } \\
\text { and have been used to estimate both contact points } \\
\text { [20] and texture [18]. }\end{array}$ \\
\hline $\begin{array}{l}\text { Peflection of the whisker near the base is measured by } \\
\text { analog occlusion of a light beam, effectively giving a } \\
\text { measurement of bending moment [25]. }\end{array}$ \\
interrupter & $\begin{array}{l}\text { A CCD camera records deformation of the whisker as } \\
\text { it contacts an object, allowing estimation of both } \\
\text { contact location and object stiffness [38]. }\end{array}$ \\
\hline \hline
\end{tabular}

which follows from the first, is that the extent of lateral slip depends on the coefficient of friction, which, in general, is not known and can vary greatly from object to object.

Both Kaneko et al. and Clements and Rahn [19], [37] independently addressed the problem of lateral slip by sensing it, and then, actively reorienting the plane of rotation until lateral slip is eliminated. However, this method has serious drawbacks: it requires adjustment of actuator orientation to keep the whisker oriented perpendicular to the object, and also, generally requires multiple rotations for each measurement of contact point location. This is awkward in practice, and infeasible when arrays of multiple whiskers are employed to contact the object.

We recently developed a method for contact point estimation that is similar to [19], but includes passive accommodation for lateral whisker slip along the object [22]. Although it was demonstrated that our method allows detailed object feature extraction with relatively modest hardware requirements, it was not examined under general contact conditions of object surface orientation, curvature, and friction. In the present manuscript, we thoroughly investigate the method in such a general setting, both analytically and experimentally.

We demonstrate the ability to quantify and passively accommodate for lateral slip of a rotating whisker in the presence of friction. This permits us to accurately determine the contact location with the object without having to reorient the rotation plane of the whisker. These new results directly enable largescale implementation on an artificial whisker array actuated with

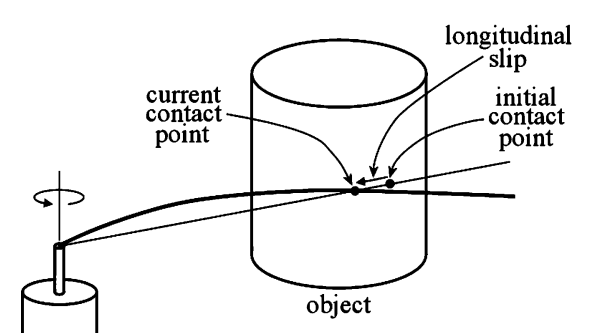

(a)

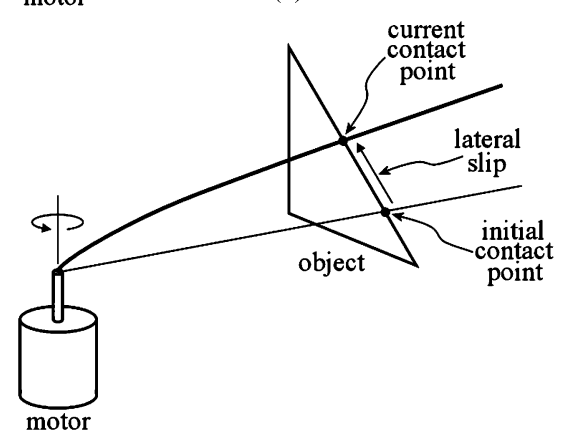

(b)

Fig. 1. There are two types of whisker slip. (a) Longitudinal slip. (b) Lateral slip. Note that although here they are depicted independently, they can in general occur simultaneously.

a single motor that may begin to mimic the ability of biological systems.

\section{Motivation: The Problem of Lateral SLIP}

One method for obtaining an estimate of 3-D object shape is to determine where in 3-D space each whisker makes initial contact with the object during the course of object exploration, and then interpolate between the resulting collection of contact points to approximate object shape [22]. If we define a cylindrical coordinate system at the base of each whisker, the goal is to estimate the value of each coordinate upon contact: $\left(r_{c}, \theta_{c}, z_{c}\right)$. We first make the assumption that the plane of rotation for each whisker is fixed at a given height $z_{c}$. The variable $\theta_{c}$ can easily be found by denoting the angle at which the moment or rate of moment change measured at the base becomes nonzero or crosses a threshold (assuming that any inertial effects will be negligible or filtered out). Finally, we are left with the task of estimating $r_{c}$-a process we will refer to as radial distance extraction.

As noted by Kaneko et al., the rotational compliance of a flexible rod rotated against an "edged" or "point" object increases as the distance to the object increases; in fact, if the rod is cylindrical in shape, compliance is directly proportional to object distance [19]. A minor complication known as longitudinal slip occurs when the point of contact moves along the length of the whisker, e.g., when the object is not a sharp edge, but instead has a finite curvature in the plane of rotation (longitudinal curvature), as depicted in Fig. 1(a). The result is a decrease in the resulting rotational compliance and underestimation of contact distance. However, Kaneko et al. also showed that (in the absence of lateral slip; discussed next) the decreased 
compliance will be negligibly small unless the surface has extremely low curvature or contact occurred very close to the whisker base [19].

A more significant complication arises when the object surface is slanted relative to the plane of whisker rotation, as shown in Fig. 1(b). In such cases, a phenomenon called lateral slip occurs in which the point of contact slides along the periphery of the object, the whisker bends outside the plane of rotation, and the resulting compliance is greater than would have occurred without slip, thus leading to an overestimation of contact distance. Techniques developed independently by Kaneko et al. [19] and Clements and Rahn [37] involve sensing the lateral slip and actively reorienting the plane of rotation until the lateral slip is eliminated, thus permitting straightforward calculation of contact point location. The problem with this approach when implemented on an array of robotic whiskers is that each whisker requires an individual motor to tilt its plane of rotation. The additional size, cost, and complexity requirements make such a solution impractical, and encourages an alternative approach.

In the present paper, we develop a model for radial distance extraction that senses lateral slip but then passively accounts for its presence through a 2-D modification of the compliance rule found by Kaneko et al. [19] to extract radial object distance. The need to adjust the plane of rotation is effectively eliminated as long as a reasonable estimate can be made for the friction coefficient between the whisker and the surface. We quantify analytically how the accuracy of the model degrades for differing friction conditions and object lateral curvatures, and suggest movement strategies to mitigate these inaccuracies. The model is experimentally validated, substantiating the reliability of the analytical results. Finally, we suggest a simple array design capable of actuating an arbitrary number of robotic whiskers with a single motor. In summary, the model demonstrates that distance extraction can be performed even in the presence of lateral slip and friction, to permit reliable and efficient overall shape extraction with a robotic whisker array.

\section{Distance Extraction In the PRESEnce OF LATERAL SLIP}

\section{A. Object Contact Along the Whisker Without Lateral Slip}

The whisker is modeled as a straight, flexible beam rotating with a fixed center of rotation and at a constant velocity. At some point, the whisker comes into contact with an object, at which time the task of finding the distance from the whisker base to contact point arises. We assume that the object is rigid and that the point of contact is fixed, discrete, and exists somewhere along the length of the whisker (as opposed to the tip, a condition that will be discussed later). In the case that slip is negligible, the configuration of the whisker can be described in two dimensions, as shown in Fig. 2 .

Because only a small deflection is needed to sense the rotational compliance, classical linear beam theory can be used. The whisker rotates by a small pushing angle $\theta$ against an object at radial distance $d_{x}$, imposing a deflection $d_{y}$. The resulting bending moment $m_{z}$ at the base of the whisker is measured by a torque sensor. Under these conditions, Kaneko et al. [19]

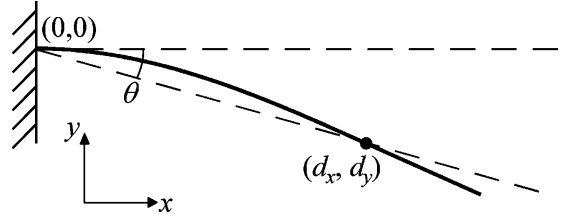

Fig. 2. Geometry of whisker deflection under no slip.

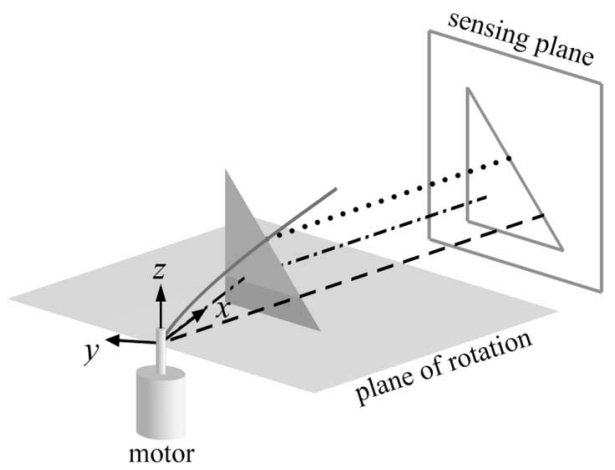

Fig. 3. A 3-D perspective view of the sensing plane.

showed that the rotational compliance $k_{\theta}$ is directly proportional to object distance, and can be expressed as:

$$
d_{x}=C k_{\theta}
$$

where $C=3 E I, E$ is the elastic modulus and $I$ is the area moment of inertia. Rotational compliance is defined as the ratio of angular displacement to bending moment at the base, allowing us to write:

$$
d_{x}=C \frac{\theta}{m_{z}} .
$$

\section{B. Factors that Influence the Magnitude of Lateral Slip}

When orientation of the object is not perpendicular to the plane of rotation at the contact point, lateral slip may occur, in which case the contact point will drag along the periphery of the object, as shown in Fig. 1(b). The resulting rotational compliance will be greater than when lateral slip does not occur, causing (2) to overestimate $d_{x}$.

Quantifying lateral slip is aided by analysis of the sensing plane [19], illustrated in Fig. 3. The sensing plane is an imaginary 2-D projection of the region where the whisker is touching the object, coplanar to the $y-z$ plane (for small $\theta$ ) and intersecting the contact point. In Fig. 3, the dashed line indicates the initial contact point, the dotted line indicates the current contact point, and the dash-dotted line indicates where the whisker would intersect the sensing plane were it not obstructed by the surface--the so-called "virtual point" [19]. A basic geometrical analysis of the relationships between variables in the sensing plane is sufficient to derive a modified form of (2) that accounts for the lateral slip. We will derive this modified equation in Section III-C.

There are specific parameters at the region of contact that affect if and how lateral slip occurs. Those parameters are as follows: 


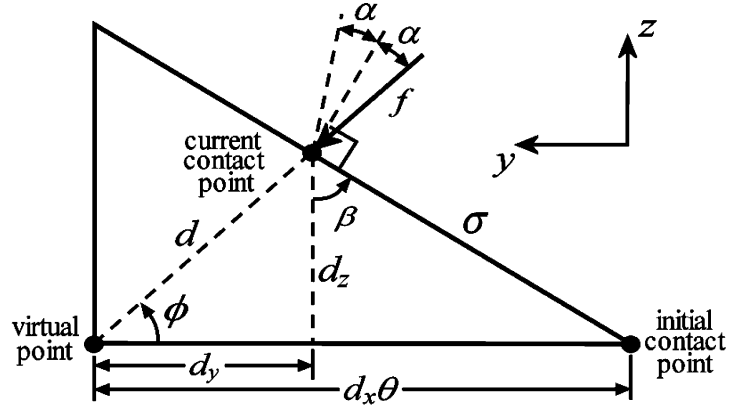

Fig. 4. A geometrical description of the sensing plane.

1) the slope of the object surface at the contact point within the sensing plane;

2) the curvature of the object surface at the contact point within the sensing plane. In analogy to the term lateral slip, we refer to this quantity as lateral curvature; and

3) the frictional properties between the whisker and the object.

In the results that follow, we will develop an equation for distance extraction that directly takes into account the slope of the surface at the contact point in the sensing plane. It will be shown that the lateral curvature has only a mildly detrimental impact on distance extraction in most cases as long as $\theta$ is sufficiently small. Friction can present some difficulties, although there are ways to address them, which will be discussed.

\section{Accounting for Lateral Slip}

In the derivation that follows, we assume that lateral slip occurs in the absence of significant concurrent longitudinal slip (as depicted in Fig. 3), the implications of which are addressed in Section VII. We also assume that the lateral curvature is zero (i.e., the surface is locally flat), and that traditional Coulomb friction exists between the whisker and the surface. Because this is a quasi-static analysis, any possible discrepancies between static and kinetic friction coefficients are assumed to be negligible.

Fig. 4 defines the relevant variables projected on the sensing plane. It geometrically predicts the slip distance $\sigma$ of the whisker along the object, depending on the local surface slope $\beta$ in the sensing plane and the friction cone angle $\alpha$. Slip will not occur when $\alpha \geq \beta$ because in this case no movement of the contact point is required to keep the contact force $f$ within or on the boundary of the friction cone, and the resulting slip angle $\phi$ will be zero. Conversely, when $\alpha<\beta$, the contact point will slip just enough to enable static equilibrium, resulting in a contact force angle of $\beta-\alpha$.

Summarizing, we have

$$
\text { if }\left\{\begin{array}{l}
\alpha \geq \beta, \quad \phi=0 \\
\alpha \leq \beta, \quad \phi=\beta-\alpha
\end{array}\right.
$$

which can be expressed more compactly as

$$
\phi=\max (0, \beta-\alpha) .
$$

Notice that the overall linear deflection $d$ is now split into two components: $d_{z}$ and $d_{y}$. This means that to sense lateral slip, the torque sensor at the base of the whisker must be equipped to measure out-of-plane bending of the whisker, that is, bending moment $m_{y}$. For small angles, the distance between the virtual point and the initial contact point is simply the contact distance $d_{x}$ multiplied by $\theta$. Following the geometry of Fig. 4, it can be shown that the slip distance $\sigma$ along the object is

$$
\sigma=\frac{d_{x} \theta}{\sin \beta+\cos \beta \cot \phi} .
$$

Using this model of lateral slip, we seek an equation analogous to (2) that will allow determination of the distance $d_{x}$ based on known and measurable variables: $E, I, \theta, m_{z}$, and $m_{y}$. Analysis of Fig. 4 along with application of some basic cantilever beam analysis (see Appendix A) yields the following result:

$$
d_{x}=C \frac{\theta}{m_{z}+m_{y} \tan \beta} .
$$

Unfortunately, there is no way to reliably estimate the surface slope $\beta$ in the presence of unknown friction. The most straightforward concession that can be made to arrive at a solution is to assume or estimate some nominal friction coefficient $\mu_{\text {est }}$. First, we note that

$$
\alpha_{\text {est }}=\tan ^{-1}\left(\mu_{\text {est }}\right)
$$

and that $\phi$ can be directly measured as

$$
\phi=\tan ^{-1}\left(\frac{m_{y}}{m_{z}}\right) .
$$

If we now assume that $\alpha_{\text {est }} \leq \beta_{\text {est }}, \beta_{\text {est }}$ can easily be computed using (3):

$$
\beta_{\mathrm{est}}=\phi+\alpha_{\mathrm{est}} .
$$

Replacing the unknown surface slope $\beta$ from (6) with $\beta_{\text {est }}$ yields

$$
d_{x}=C \frac{\theta}{m_{z}+m_{y} \tan \beta_{\mathrm{est}}} .
$$

Note that (10) is reliable even when $\alpha_{\text {est }}>\beta_{\text {est }}$ [contrary to the assumption made in (9)] because in this case, $m_{y}=0$ and the term $m_{y} \tan \beta_{\text {est }}$ becomes zero as well.

One obvious situation to consider is that of frictionless contact, resulting in $\beta_{\text {est }}=\phi$ and thus

$$
d_{x}=C \frac{\theta}{m_{z}+m_{y}^{2} / m_{z}}
$$

which can alternatively be written as

$$
d_{x}=C \frac{\theta}{|m|} \cos \phi
$$

where $|m|=\sqrt{m_{z}^{2}+m_{y}^{2}}$ as in [22].

In the following section, the two most significant potential sources of distance extraction error are addressed analytically, namely, discrepancy between $\alpha_{\text {est }}$ and the true $\alpha$, and lateral curvature of the object surface. 


\section{Potential Sources of Distance Extraction ERror}

\section{A. Unknown Friction Coefficient}

Distance extraction using (10) requires an estimate of the friction coefficient between the whisker and the surface. In order to characterize how discrepancy between $\alpha_{\text {est }}$ and the true $\alpha$ results in distance extraction error, we return to the lateral slip model of Fig. 4 to obtain (see Appendix A):

$$
\begin{aligned}
& m_{z}=\frac{C \theta}{d_{x}}\left(\frac{1}{\tan \beta \tan \phi+1}\right) \\
& m_{y}=\frac{C \theta}{d_{x}}\left(\frac{1}{\tan \beta+\cot \phi}\right) .
\end{aligned}
$$

Equations (13) and (14) along with (4) define how $m_{z}$ and $m_{y}$ will increase as the whisker rotates against a slanted surface as a function of $\alpha$ and $\beta$. Thus, assuming some estimate of friction cone angle $\alpha_{\text {est }}$, we can numerically compute the surface slope $\beta_{\text {thresh }}$ at which at a given percentage of distance extraction error (an "error threshold") is incurred for a range of actual friction cone angles $\alpha$. $\beta_{\text {thresh }}$ can in principle range between $0^{\circ}$ (vertical) and $90^{\circ}$ (horizontal). The larger $\beta_{\text {thresh }}$ is, the more reliable the distance extraction is in the presence of a complex and/or arbitrarily oriented object.

Fig. 5(a)-(c) shows the relation between $\beta_{\text {thresh }}$ and $\alpha$ for $\alpha_{\text {est }}=0^{\circ}, 10^{\circ}$, and $20^{\circ}$, respectively. The surface slope at $1 \%, 5 \%$, and $10 \%$ error thresholds for distance extraction using (2) and (10) are represented by dashed lines and the solid lines, respectively. Naturally, as the error threshold increases, so does $\beta_{\text {thresh }}$ for both distance extraction equations. However, Fig. 5(a) shows that (10) is always equally or more accurate than (2) when $\alpha_{\text {est }}=0^{\circ}$, with the relative advantage becoming progressively more significant with increasing error threshold level. The advantage of (10) over (2) is even more pronounced when a reasonable guess can be offered for $\alpha_{\text {est }}$. So long as $\alpha_{\text {est }}$ falls within a moderate range of $\alpha$, distance extraction will be accurate even for steep surface slopes. For example, Fig. 5(b) tells us that if $\alpha_{\text {est }}=10^{\circ}$, distance extraction will be accurate to within $5 \%$ so long as $\alpha$ ranges between $8.2^{\circ}$ and $11.7^{\circ}$ and $\beta \leq 60^{\circ}$, or to within $10 \%$ so long as $\alpha$ ranges between $7.8^{\circ}$ and $12.3^{\circ}$ and $\beta \leq 70^{\circ}$.

\section{B. Lateral Curvature of the Object Surface}

Equation (10) assumes that the surface being contacted has a lateral curvature of zero. Although this at first may seem to be a restrictive assumption, one must consider the effect of curvature within the scale of the contact distance. The radius of curvature $r_{\kappa}$ scaled relative to the contact distance $d_{x}$, defines the normalized lateral curvature as

$$
\kappa_{n}=\frac{d_{x}}{r_{\kappa}}
$$

and thus, the normalized radius of curvature as $r_{n}=1 / \kappa_{n}$. The smaller $\kappa_{n}$ is, the flatter the surface effectively is. Note, however, that the effect of curvature on distance extraction must be considered in the context of three additional variables: $\theta$ and $\alpha$,

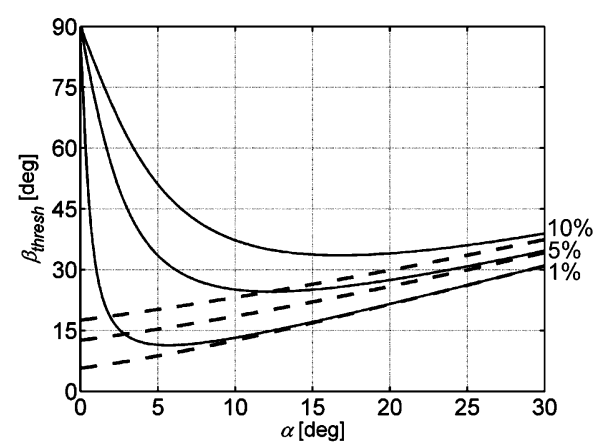

(a)

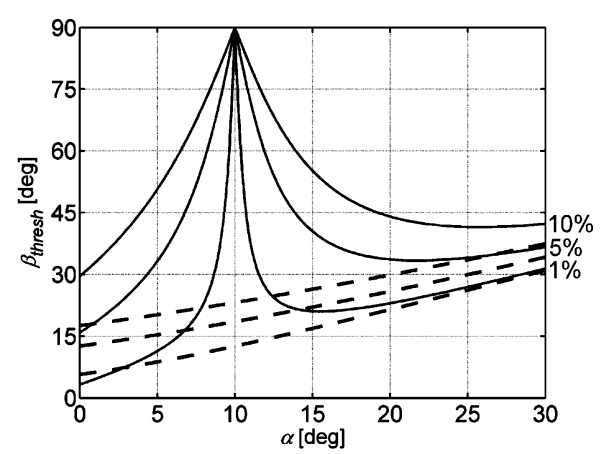

(b)

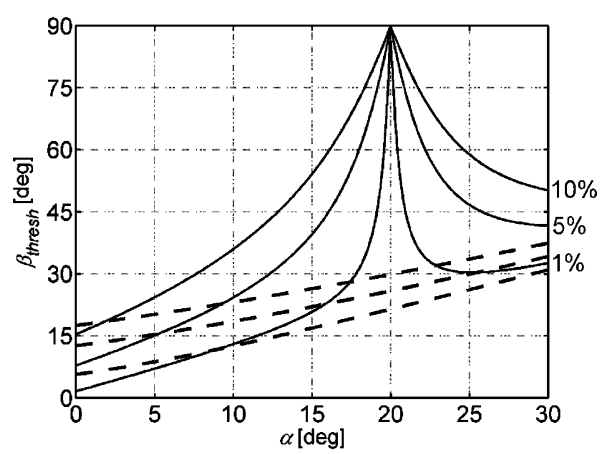

(c)

Fig. 5. Error threshold analysis for maximum allowable surface slope given particular deviations between $\alpha$ and $\alpha_{\text {est }}$ with (a) $\alpha_{\text {est }}=0^{\circ}$. (b) $\alpha_{\text {est }}=10^{\circ}$. (c) $\alpha_{\text {est }}=20^{\circ} . \beta_{\text {thresh }}$ is the surface slope at which at a given percentage of distance extraction error is incurred for a range of actual friction cone angles $\alpha$. Dashed lines represent $\beta_{\text {thresh }}$ calculated using (2). Solid lines represent $\beta_{\text {thresh }}$ calculated using (10).

as defined earlier, and also, $\beta_{0}$ - the surface slope in the sensing plane at the initial contact point. To understand the interplay between these variables, we may refer to error threshold plots similar to those in Fig. 5, but now with $r_{n}$ as the independent variable (see Appendix B for derivation).

Fig. 6(a)-(c) plots the maximum allowable $\beta_{0}\left(\beta_{\text {thresh }}\right)$ as a function of $r_{n}$ that keeps distance extraction error under $1 \%, 5 \%$, and $10 \%$, respectively. Each plot shows these relationships for three different pushing angles: $\theta=$ $1^{\circ}, 3^{\circ}$, and $5^{\circ}$. As expected, $\beta_{\text {thresh }}$ always increases with increased error threshold and with increased $r_{n}$ (surface flatness).

Interestingly, Fig. 6 illustrates that friction has an overall negligible impact, while in contrast, increasing $\theta$ has a significantly 


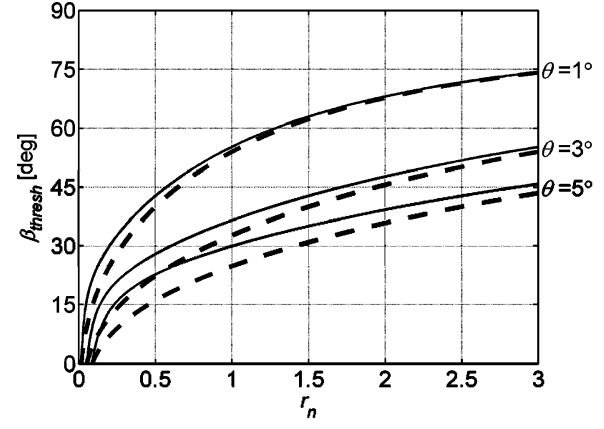

(a)

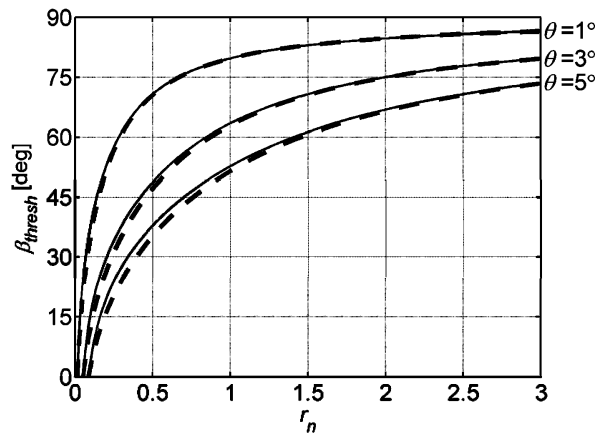

(b)

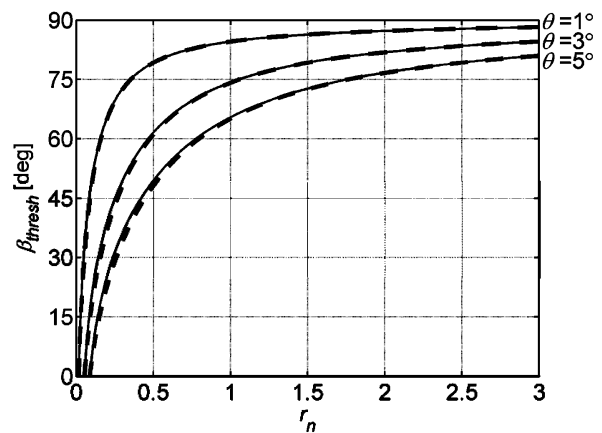

(c)

Fig. 6. Error threshold analysis for maximum allowable surface slope given particular normalized lateral radii of curvature and (a) $1 \%$ error. (b) $5 \%$ error. (c) $10 \%$ error. Dashed lines are for contact with $\alpha=\alpha_{\text {est }}=0^{\circ}$, and solid lines are for contact with $\alpha=\alpha_{\mathrm{est}}=20^{\circ}$.

detrimental impact. The reason for this goes back to the issue of scale - a larger $\theta$ will result in a larger slip relative to any given $r_{n}$, and thus, more deviation from the assumption of a flat surface. As a practical issue, the benefit of using a small $\theta$ to guard against lateral curvature must be balanced against the decreased reaction torque at the whisker base that will result. Generally speaking, $\theta$ should be chosen to be as small as possible while still ensuring a sufficient reaction torque to allow accurate distance extraction when contact occurs near the whisker tip.

Overall, Fig. 6 shows that the consequence of lateral curvature is relatively mild. For example, given a significantly curved surface with $r_{n}=1$ and a realistic pushing angle of $\theta=3^{\circ}$, a $\beta_{0}$ of $63^{\circ}$ will result in $5 \%$ distance extraction error and a $\beta_{0}$ of $74^{\circ}$ will result in $10 \%$ error. Increasing $\theta$ to $5^{\circ}$ would decrease the maximum $\beta_{0}$ to $52^{\circ}$ and $65^{\circ}$, respectively.

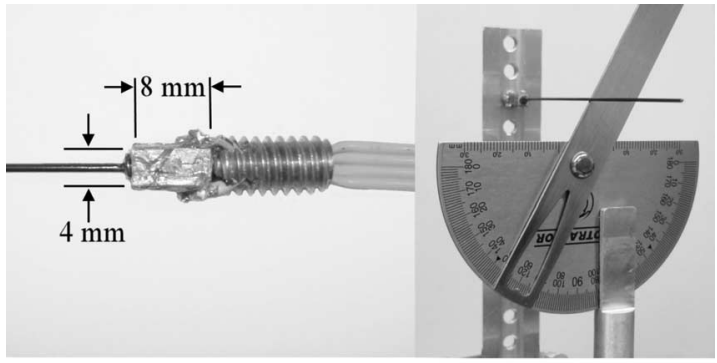

(a)

(b)

Fig. 7. (a) Whisker follicle. (b) Experimental setup for model validation experiment.

\section{MODEL VALIDATION}

Experimental validation of the lateral slip model was performed. The artificial whisker used for these experiments had two components: a flexible beam (the "vibrissa") and a two-axis torque sensor (the "follicle") [see Fig. 7(a)].

The vibrissa is a straight superelastic Nitinol wire, $1 \mathrm{~mm}$ in diameter and $10 \mathrm{~cm}$ in length, with a small setscrew attached at the base. The relatively thick wire diameter was chosen to ensure a strong torque signal to sense small lateral deflections; generally, much thinner whiskers can be used. The setscrew allows different size and shape vibrissa to be easily interchanged within the follicle. The follicle is a small aluminum block $(4 \times 4 \times 8 \mathrm{~mm})$ with the center tapped to match the setscrew at the base of the vibrissa. Each of the four faces of the follicle is fitted with a strain gage, allowing independent measurement of $m_{z}$ and $m_{y}$, and the follicle itself is attached to a larger setscrew to allow easy connection to an array. For these experiments, the array was simply a vertically oriented aluminum bar with a series of threaded holes, which allowed whiskers to be configured in a single column (see Section VI). The array was attached to an AC servomotor at the base for actuation. The most important, but not essential, feature of the present design is that it positions the base of the vibrissae (and the tips of their follicles) at the center of rotation, thus conforming to the model as expressed in (13) and (14). Equations (13) and (14) could be modified to account for noncenter rotation (similar to [20]), but that was not tested in the present experiments.

The experiment involved rotating the whisker against a slender stainless steel bar [see Fig. 7(b)] at a variety of surface slopes: from $\beta=0^{\circ}$ (vertical) to $75^{\circ}$ (nearly horizontal) in $5^{\circ}$ increments (similar to [20]), and at two different speeds: $\dot{\theta}=$ $10 \%$ and $90 \%$ s. Signals from the follicle strain gages were first calibrated from voltage to moment. The $m_{y}$ component was calibrated by rotating the whisker against the bar with $\beta=0^{\circ}$ at a radial distance of $d_{x}=5 \mathrm{~cm}$. Ten whisks were performed at both $\dot{\theta}=10 \%$ and $90 \%$ s. The location at which the whisker gently touched the bar was defined as $\theta=0^{\circ}$. Whisker trajectories were chosen to ensure a smooth acceleration to the maximum velocity. For $\dot{\theta}=10 \%$, the whisker was rotated through a range of $-3^{\circ}$ to $12^{\circ}$, while for $\dot{\theta}=90^{\circ} / \mathrm{s}$, an expanded range of $-15^{\circ}$ to $20^{\circ}$ was used. All data were filtered at $800 \mathrm{~Hz}$, sampled at $2000 \mathrm{~Hz}$, and passed through a zero-phase digital filter with a cutoff frequency of either $5 \mathrm{~Hz}$ (for $\dot{\theta}=10^{\circ} / \mathrm{s}$ ) or $45 \mathrm{~Hz}$ 

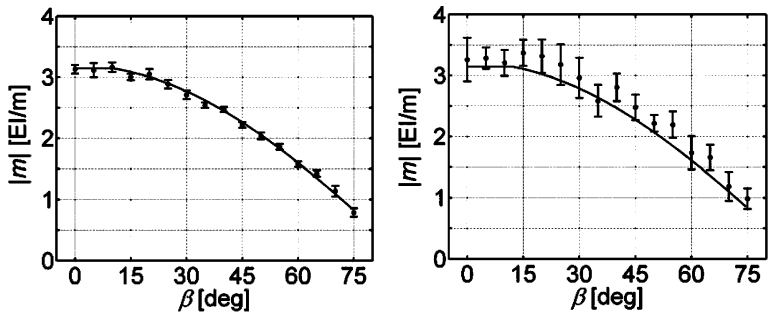

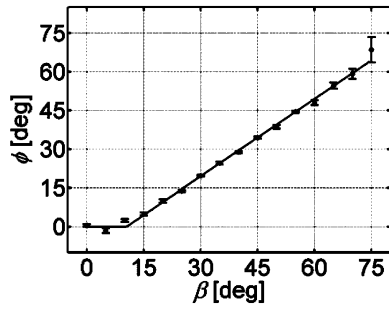

(a)
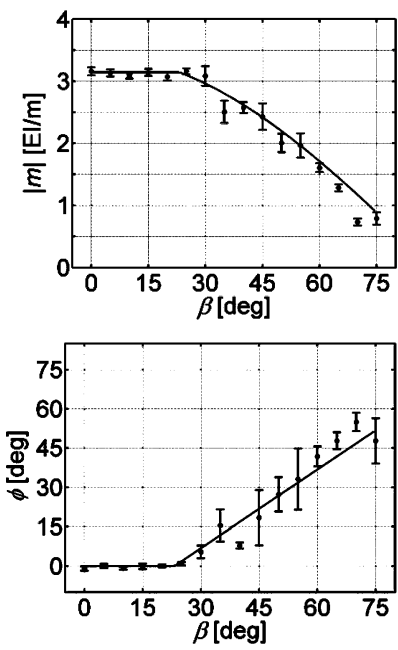

(c)

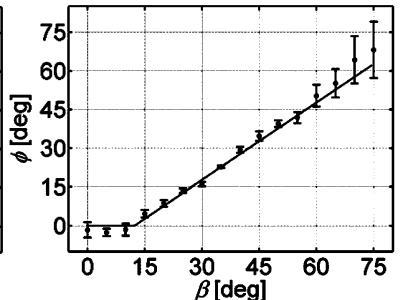

(b)
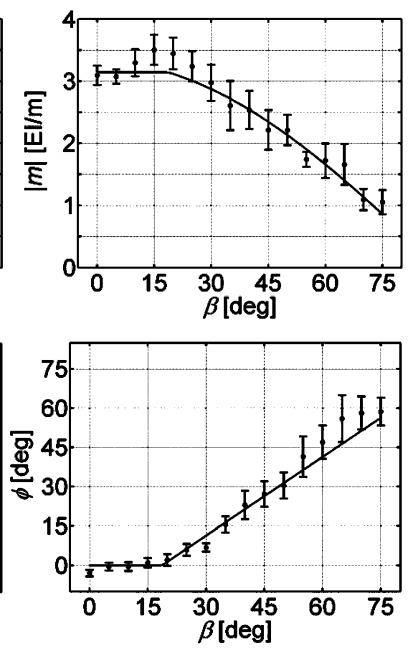

(d)
Fig. 8. Experimental results for stainless steel surface and (a) $\dot{\theta}=10^{\circ} / \mathrm{s}$, (b) $\dot{\theta}$ $=90^{\circ} / \mathrm{s}$, and for sandpaper surface with (c) $\dot{\theta}=10^{\circ} / \mathrm{s}$, (d) $\dot{\theta}=90^{\circ} / \mathrm{s}$. Error bars are standard deviations. Solid lines are LMS fits, calculated as described in the text.

(for $\dot{\theta}=45^{\circ} / \mathrm{s}$ ). Using (2) along with a pushing angle $\theta=3^{\circ}$, we arrive at a calibration factor between voltage and moment $m_{y}$. The follicle was then rotated $90^{\circ}$ and the calibration process repeated for $m_{z}$.

Experimental data were gathered in the same way as the calibration process, but for varying $\beta$. For each $\beta$, the plane of the bar was perpendicular to the orientation of the whisker at $\theta=0^{\circ}$. Fig. 8(a) and (b) plots $|m|$ and $\phi$ versus $\beta$ for the two different whisking velocities. Units for $|m|$ are $E I /$ meters, that is, equivalent to $E I \cdot \kappa$ since $\kappa=m / E I$. Fig. 8(c) and (d) is the same as Fig. 8(a) and (b), except that a rough surface was tested by covering the object surface with 180 grit (fine) sandpaper. The sandpaper was replaced for each $\beta$ to prevent wear from affecting the results. Least-mean-square (LMS) fits were performed on the experimental data sets. Specifically, LMS fits were performed on the sum of the squares of (13) and (14) with $\alpha_{\text {est }}$ as the free parameter. Absolute value of the moment was then calculated as $|m|=\sqrt{m_{z}^{2}+m_{y}^{2}}$, and $\phi$ was calculated from (8).

Fig. 8(a) demonstrates that an excellent fit between model and experiment was obtained for whisking against the steel rod at $10^{\circ} \%$, for an estimated value of friction cone angle $\alpha_{\text {est }}=10.5^{\circ}$. At $90^{\circ} / \mathrm{s}$ [see Fig. 8(b)], the data again conforms well to the model, the fit yielding $\alpha_{\mathrm{est}}=12.3^{\circ}$, but the measurement variability is significantly higher. The increased variability is likely due to dynamic effects, namely, vibrations, starting to become significant, though this was not confirmed.

Fig. 8(c) and (d) illustrates the phenomenon of stick-slip friction in which the whisker irregularly switches between periods of no slip, smooth slip, and sudden, abrupt slip. This led to a high level of variability in the data obtained at $\dot{\theta}=10 \%$, but had a smaller effect at $\dot{\theta}=90^{\circ}$ s. The difference is likely due to the well-known sensitivity of stick-slip behavior to velocity. The fits to $\dot{\theta}=10^{\circ} \%$ s and $90^{\circ} / \mathrm{s}$ yielded $23.3^{\circ}$ and $18.7^{\circ}$, respectively, for $\alpha_{\text {est }}$.

\section{ARRAY DESIGN}

Although the results presented here have specifically addressed radial distance extraction with a single whisker, they are particularly relevant to implementation on an array of robotic whiskers. Because the proposed method allows radial distance extraction to be performed in the presence of lateral slip, multiple whiskers can be configured into arrays wherein their relative base positions and planes of rotation are fixed. Hence, the method enables the synchronous movement of multiple whiskers against an arbitrarily shaped and oriented object to efficiently collect multiple contact points, which can be processed in parallel to extract complex surface features [22].

Because whisking often involves the rotation of multiple whiskers in close synchrony, a desirable property of a whisker array is the need for only a single motor for actuation. In [17], a servomotor is used to move a flexible membrane, through which the base region of multiple whiskers is threaded, thus allowing a $40^{\circ}$ whisking amplitude. In [20], a DC motor connects to a support plate upon which multiple whiskers are attached at varying angles. As mentioned in Section $\mathrm{V}$, the present model requires that each whisker rotate about its cantilevered base-where the moment sensor resides - necessitating a different design than [17] and [20]. Our solution was to mill an aluminum cylinder down to a slender plank except for the region at the base, where a hole is drilled for attachment to an AC servomotor. Several holes were then drilled through the plank and threaded for attachment of the whiskers. Fig. 9 shows the entire array along with four whiskers, similar to the one used in the previous experiments [see Fig. 7(a)].

In order to actuate multiple columns of whiskers, a simple solution is to use a classic multibar linkage system, such as the one used in [28], thus allowing for the actuation of an arbitrarily sized grid of whiskers, still using only a single motor. 


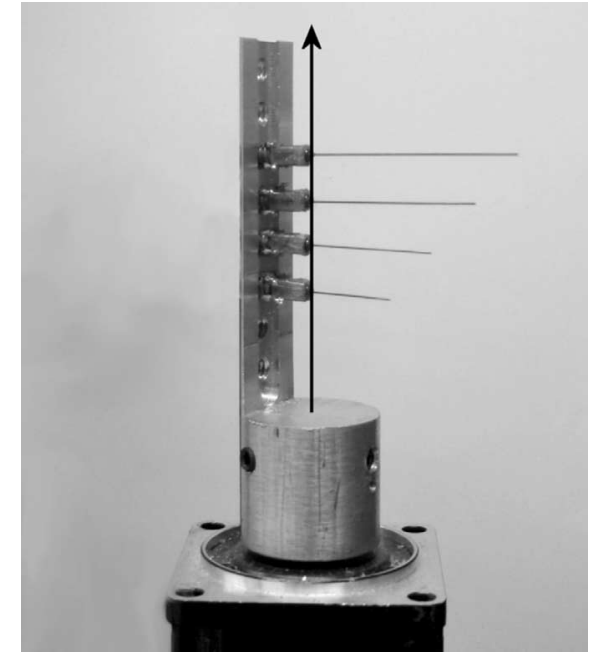

Fig. 9. Array design for a single column of whiskers.

\section{DISCUSSION}

This paper has demonstrated that reorientation of the whisking plane is not necessary for reliable measurement of contact point location, thus opening the possibility for whisker arrays of simple mechanical design capable of detailed 3-D feature extraction. In order to perform accurate feature extraction with objects of arbitrary shape and orientation, a reasonable estimate of the friction coefficient between the whisker and the object is beneficial. In many cases, it may be reasonable to assume that the environment is composed of mostly smooth surfaces, in which case an estimate of $\mu_{\text {est }}=0.18\left(\alpha_{\text {est }}=10^{\circ}\right)$ should be reasonable, as indicated in by the fits in Fig. 8 (a) and (b). If that assumption is inaccurate and the surface is rough, say with $\alpha=20^{\circ}$, then Fig. 5(b) tells us that distance extraction will remain accurate to within $10 \%$ error as long as the surface slope $\beta$ is less than about $45^{\circ}$ (also depending on the normalized lateral curvature $\kappa_{n}$ and pushing angle $\theta$, as shown in Fig. 6).

If a more reliable estimate is desired, there are a few possible solutions, as follows.

1) If the orientation of the entire whisker array can be tilted along the $x$-axis of any particular whisker, whisks would be performed against the object at a variety of orientations, effectively adjusting the relative surface slope. The range of slopes for which $\phi=0^{\circ}$ would be equal to $2 \alpha$ (the accuracy depending on how gradually the orientation was adjusted). This essentially amounts to an in-field test of the object to determine the friction coefficient. Note that this change in orientation occurs for the entire array (not for each individual whisker) and only needs to be done when a new estimate of friction is desired.

2) Have the array explore the object, and repeat the feature extraction algorithm (the transformation of contact points into 3-D object shape, e.g., splining [22]) using a range of friction coefficients. The one that leads to the most consistent contact point positions and thus the smoothest surface is kept.
3) Have the array whisk against the object at a variety of array orientations. All the contact points sampled are given a confidence measure related to $\phi$, such that points with large $\phi$ are ignored or have less influence on the feature extraction algorithm.

Methods 1 and 2 assume that the friction coefficient is constant over the entire object, while method 3 does not.

Another problem involves the occurrence of stick-slip in which the coefficient of static friction is significantly greater than that of kinetic friction (which the model assumes are the same). However, we have shown experimentally that stick-slip is reduced by whisking quickly (e.g., $90^{\circ} / \mathrm{s}$ ), a behavior that is also desirable for the sake of sensing an object quickly, as rats are able to do.

Other potential sources of distance extraction error include the following.

1) Simultaneous longitudinal and lateral slip: The analysis performed in this paper assumes that no longitudinal slip occurs along with the lateral slip. However, this assumption is not always valid. Consider the case in which the object shown in Fig. 3 is tilted by an angle $\psi$ either toward or away from the motor instead of oriented vertically. This could cause simultaneous lateral and longitudinal slip, depending on the friction conditions and particular values of $\beta$ and $\psi$. Additional analyses are required to quantify the effect on distance extraction, but it should be small unless both $\beta$ and $\psi$ are large.

2) Object compliance: A solution is offered in [19] in which two whisks are performed on the object from two different distances, and the difference in whisker compliance reveals both the contact distance and a measure of the object compliance.

3) Multipoint contact: This should be rare even for complex objects because it would require two distinct point along the object's surface to closely line up along the initial contact orientation of the whisker. As the pushing angle $\theta$ becomes smaller, this situation becomes increasingly unlikely.

4) Moving objects: Most environments are static on the temporal scale over which exploration is likely to occur. Also, if the environment is moving, its velocity is often negligible relative to the whisking speed $\dot{\theta}$. Because the whiskers have very little mass, they can move very rapidly (in rats, up to $1500^{\circ} / \mathrm{s}[3]$ ).

One final issue involves contact occurring at the whisker tip. Such cases may result in decreased rotational compliance, causing (6) to estimate $d_{x}$ to be greater than the whisker length. The solution is to simply give $d_{x}$ a threshold equal to the whisker length [22]. It is also worth noting that regions of an object that are concave with respect to the plane of whisker rotation can only be sampled through tip contact if the whisker is straight. Tip contact is thus actually desirable for two reasons: it potentially helps provide a very precise measurement of contact distance (since the whisker length is exactly known), and it allows concave regions of an object to be sampled.

We point out that the method presented here is not limited to the case of a cylindrical whisker. In general, moment can be 
related to pushing angle by a monotonically decreasing stiffness function that depends on the shape and elastic modulus of the whisker [22]. This allows us to write (12) in the more general form:

$$
d_{x}=\nu(\theta,|M|) \cos \phi
$$

where $\nu(\theta,|M|)$ is the distance extraction equation for the whisker in the case of no lateral slip and $\phi$ is from (8). For the case of a conical beam, which is a good approximation for a rat whisker [22], we have:

$$
d_{x}=\frac{C \theta L}{C \theta+|M| L} \cos \phi,
$$

where $L$ is the base-to-tip whisker length.

\section{CONCLUSION}

We have demonstrated the effectiveness of a method to measure contact point location with an artificial whisker in the presence of significant lateral slip and surface friction. The method requires only that the whisker be equipped with a two-axis torque sensor at the base, and works best when a reasonable estimation for the object's friction coefficient is available. The biggest advantage of this method is that it does not require that each whisker can independently adjust its plane of rotation, and is thus very amenable to implementation on a large-scale array. The effects of inaccurately estimating surface friction and lateral curvature were quantified. The next step is to construct a large (perhaps $5 \times 5$ ) whisker array, and show that it can be used to quickly and consistently extract complex object features using only a small number of whisks.

\section{APPENDIX}

\section{A. Derivation of Distance Extraction Model (6)}

Equation (2) provided a relation between bending moment at the whisker base $m_{z}$, the radial distance $d_{x}$, and the pushing angle $\theta$ :

$$
d_{x}=C \frac{\theta}{m_{z}} .
$$

Now, referring to Fig. 2, it is clear that $\theta=\tan \left(d_{y} / d_{x}\right)$. For small angles, $\theta=d_{y} / d_{x}$, which we plug into (2) to obtain:

$$
m_{z}=C \frac{d_{y}}{d_{x}^{2}} .
$$

Note that this equation holds when lateral slip occurs (see Fig. 4) because it simply relates moment in the $z$-direction to radial distance $d_{x}$ and deflection in the $y$-direction, none of which are affected by independent deflection of the whisker in the $z$-direction $\left(d_{z}\right)$. By symmetry, the same linear scaling between $m_{z}$ and $d_{y}$ also holds for $m_{y}$ and $d_{z}$ :

$$
m_{y}=C \frac{d_{z}}{d_{x}^{2}} .
$$

Furthermore, from Fig. 4, it is clear that

$$
\tan \beta=\frac{d_{x} \theta-d_{y}}{d_{z}}
$$

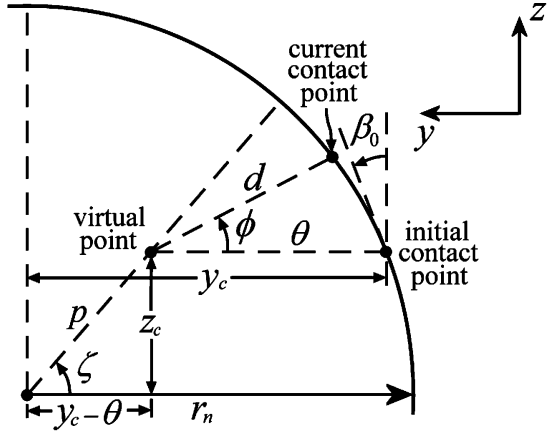

Fig. 10. Geometrical description of the sensing plane with lateral object curvature.

Combining (18)-(20) and solving for $d_{x}$ yields:

$$
d_{x}=C \frac{\theta}{m_{z}+m_{y} \tan \beta} .
$$

Further inspection of Fig. 4 reveals

$$
\tan \phi=\frac{d_{z}}{d_{y}} .
$$

Combining (18), (20), and (21) and solving for $m_{z}$ yields:

$$
m_{z}=\frac{C \theta}{d_{x}}\left(\frac{1}{\tan \beta \tan \phi+1}\right) .
$$

Combining (19)-(21) and solving for $m_{y}$ yields:

$$
m_{y}=\frac{C \theta}{d_{x}}\left(\frac{1}{\tan \beta+\cot \phi}\right) .
$$

\section{B. Derivation of Slip Behavior in Presence of Lateral Curvature}

Fig. 10 is a sensing plane diagram for the whisker rotating against a circle with initial contact point $\left(y_{c}, z_{c}\right)$ relative to the center. Of course, the object need not literally be a circle; what matters is that any point along a (2-D) surface can be uniquely characterized by a single radius of curvature. Throughout this derivation, all distance units are normalized by contact distance $d_{x}$.

We seek to characterize the performance of (6) as a function of four independent variables: $r_{n}, \theta, \alpha$, and $\beta_{0}$. That is, we need to find expressions for $m_{z}$ and $m_{y}$ as functions of these variables. The first step is to observe the conspicuous relations

$$
\left\{\begin{array}{l}
m_{z}=d \cos \phi \\
m_{y}=d \sin \phi .
\end{array}\right.
$$

This immediately shifts our task to finding expressions for $d$ and $\phi$. As before, the whisker will laterally slip only if the initial contact angle is less than the friction cone angle, in which case the contact force will remain at the edge of the friction cone. Because the surface curvature now allows the contact angle to change, we modify (3) by replacing $\beta$ with the angle $\zeta$ (defined in Fig. 10) and noting that the constraint on slipping now depends on $\beta_{0}$, the surface slope in the sensing plane at the 
initial contact point:

$$
\text { if } \begin{cases}\alpha \geq \beta_{0}, & \phi=0 \\ \alpha \leq \beta_{0}, & \phi=\zeta-\alpha .\end{cases}
$$

Equation (23) can be reduced to

$$
\phi=\max (0, \zeta-\alpha)
$$

because $\zeta>\beta_{0}$ for any finite pushing angle $\theta$.

To solve for $\phi$ requires that we express $\zeta$ in terms of the independent variables; so, again examining Fig. 10, we find:

$$
\zeta=\tan ^{-1}\left(\frac{z_{c}}{y_{c}-\theta}\right) .
$$

The denominator of (25) may seem puzzling unless it is recalled that each term has been normalized by contact distance $d_{x}$, so that $y_{c}$ and $\theta$ are both unitless. To find expressions for $y_{c}$ and $z_{c}$, we again refer to Fig. 10 to find

$$
r_{n}^{2}=y_{c}^{2}+z_{c}^{2}
$$

and

$$
\tan \beta_{0}=\frac{z_{c}}{y_{c}}
$$

Equations (26) and (27) are combined to yield:

$$
\left\{\begin{array}{l}
y_{c}=\frac{r_{n}}{\sqrt{\tan ^{2} \beta_{0}+1}} \\
z_{c}=\frac{r_{n} \tan \beta_{0}}{\sqrt{\tan ^{2} \beta_{0}+1}} .
\end{array}\right.
$$

Inserting (28) into (25), we obtain

$$
\zeta=\tan ^{-1}\left(\frac{r_{n} \tan \beta_{0}}{r_{n}-\theta \sqrt{\tan ^{2} \beta_{0}+1}}\right)
$$

which can be inserted into (24) to find $\phi$.

Now seeking an expression for $d$, we apply the Pythagorean Theorem to Fig. 10:

$$
r_{n}^{2}=(p \cos \zeta+d \cos \phi)^{2}+(p \sin \zeta+d \sin \phi)^{2} .
$$

Solving (30) for $d$

$$
d=\sqrt{r_{n}^{2}-\frac{p^{2}}{2}(1-\cos (2(\zeta-\phi)))}-p \cos (\zeta-\phi)
$$

where $p$ can be found using the Pythagorean Theorem:

$$
p=\sqrt{\left(y_{c}-\theta\right)^{2}+z_{c}^{2}} .
$$

Finally, (28), (29), (31), and (32) can be combined to express $d$ as a function of the independent variables, though we were unable to obtain a compact expression.

\section{ACKNOWLEDGMENT}

The authors would like to thank Dr. M. Peshkin for his constructive comments.

\section{REFERENCES}

[1] F. Anjum, H. Turni, P. G. H. Mulder, J. Van Der Burg, and M. Brecht, "Tactile guidance of prey capture in Etruscan shrews," in Proc. Nat. Acad. Sci. USA, Oct.31, 2006, vol. 103, pp. 16544-16549.

[2] D. Bachteler and G. Dehnhardt, "Active touch performance in the Antillean manatee: Evidence for a functional differentiation of facial tactile hairs," Zoology-Anal. Complex Syst., vol. 102, pp. 61-69, 1999.

[3] G. E. Carvell and D. J. Simons, "Biometric analyses of vibrissal tactile discrimination in the rat," J. Neurosci., vol. 10, pp. 2638-2648, 1990.

[4] G. Dehnhardt and G. Ducker, "Tactual discrimination of size and shape by a California sea lion (Zalophus californianus)," Animal Learning Behav., vol. 24, pp. 366-374, Nov. 1996.

[5] G. Dehnhardt and A. Kaminski, "Sensitivity of the mystacial vibrissae of harbor seals (Phoca-Vitulina) for size differences of actively touched objects," J. Exp. Biol., vol. 198, pp. 2317-2323, Nov. 1995.

[6] G. Dehnhardt, B. Mauck, and H. Bleckmann, "Seal whiskers detect water movements," Nature, vol. 394, pp. 235-236, Jul. 16, 1998.

[7] E. Guic-Robles, C. Valdivieso, and G. Guajardo, "Rats can learn a roughness discrimination using only their vibrissal system," Behav. Brain Res., vol. 31, pp. 285-289, 1989.

[8] G. Miller, "Society for neuroscience meeting - Bats have a feel for flight," Science, vol. 310, pp. 1260-1261, Nov. 25, 2005.

[9] R. L. Reep, C. D. Marshall, and M. L. Stoll, "Tactile hairs on the postcranial body in Florida manatees: A mammalian lateral line?," Brain Behav. Evol., vol. 59, pp. 141-154, 2002.

[10] R. L. Reep, C. D. Marshall, M. L. Stoll, and D. M. Whitaker, "Distribution and innervation of facial bristles and hairs in the Florida manatee (Trichechus manatus latirostris)," Marine Mammal Sci., vol. 14, pp. 257273, Apr. 1998.

[11] R. L. Reep, M. L. Stoll, C. D. Marshall, B. L. Homer, and D. A. Samuelson, "Microanatomy of facial vibrissae in the Florida manatee: The basis for specialized sensory function and oripulation," Brain Behav. Evolution, vol. 58, pp. 1-14, 2001.

[12] D. K. Sarko, R. L. Reep, J. E. Mazurkiewicz, and F. L. Rice, "Adaptations in the structure and innervation of follicle-sinus complexes to an aquatic environment as seen in the Florida manatee (Trichechus manatus latirostris)," J. Comparative Neurol., vol. 504, pp. 217-237, Sep. 2, 2007.

[13] N. Schulte-Pelkum, S. Wieskotten, W. Hanke, G. Dehnhardt, and B. Mauck, "Tracking of biogenic hydrodynamic trails in harbour seals (Phoca vitulina)," J. Exp. Biol., vol. 210, pp. 781-787, Mar. 1, 2007.

[14] S. B. Vincent, "The tactile hair of the white rat," J. Comput. Neurosci., vol. 23, pp. 1-38, 1913.

[15] W. I. Welker, "Analysis of sniffing of the albino rat," Behaviour, vol. 22, pp. 223-244, 1964.

[16] D. J. Krupa, M. S. Matell, A. J. Brisben, L. M. Oliveira, and M. A. L. Nicolelis, "Behavioral properties of the trigeminal somatosensory system in rats performing whisker-dependent tactile discriminations," $J$. Neurosci., vol. 21, pp. 5752-5763, Aug. 1, 2001.

[17] M. Fend, S. Bovet, H. Yokoi, and R. Pfeifer, "An active artificial whisker array for texture discrimination," in Proc. 2003 IEEE/RSJ Int. Conf. Intell. Robots Syst., Las Vegas, USA, 2003, pp. 1044-1049.

[18] J. Hipp, E. Arabzadeh, E. Zorzin, J. Conradt, C. Kayser, M. E. Diamond, and P. König, "Texture signals in whisker vibrations," J. Neurosci., vol. 95, pp. 1792-1799, 2006.

[19] M. Kaneko, N. Kanayama, and T. Tsuji, "Active antenna for contact sensing," IEEE Trans. Robot. Autom., vol. 14, no. 2, pp. 278-291, Apr. 1998.

[20] D. Kim and R. Möller, "Biomimetic whiskers for shape recognition," Robot. Auton. Syst., vol. 55, pp. 229-243, Mar. 2007.

[21] G. R. Scholz and C. D. Rahn, "Profile sensing with an actuated whisker," IEEE Trans. Robot. Autom., vol. 20, no. 1, pp. 124-127, Feb. 2004.

[22] J. H. Solomon and M. J. Hartmann, "Robotic whiskers used to sense features," Nature, vol. 443, Oct. 5, 2006.

[23] M. J. Pearson, A. G. Pipe, C. Melhuish, B. Mitchinson, and T. J. Prescott, "Whiskerbot: A robotic active touch system modeled on the rat whisker sensory system," Adaptive Behav., vol. 15, pp. 223-240, 2007.

[24] R. A. Brooks, "A robot that walks; emergent behaviors from a carefully evolved network," Neural Comput., vol. 1, pp. 253-262, 1989.

[25] S. Hirose, S. Inoue, and K. Yoneda, "The whisker sensor and the transmission of multiple sensor signals," Adv. Robot., vol. 4, pp. 105-117, 1990.

[26] D. Jung and A. Zelinsky, "Whisker based mobile robot navigation," in Proc. 1996 IEEE/RSJ Int. Conf. Intell. Robots Syst., Osaka, Japan, pp. 497504. 
[27] M. Fend, H. Yokoi, and R. Pfeifer, "Optimal morphology of a biologicallyinspired whisker array on an obstacle-avoiding robot," in Proc. 7th Eur. Conf. Artif. Life, Dortmund, Germany, 2003, pp. 771-780.

[28] A. E. Schultz, J. H. Solomon, M. J. Peshkin, and M. J. Hartmann, "Multifunctional whisker arrays for distance detection, terrain mapping, and object feature extraction," in Proc. 2005 IEEE Int. Conf. Robot. Autom., Barcelona, Spain, 2008, pp. 2588-2593.

[29] V. V. Hafner, M. Fend, P. König, and K. P. Kording, "Predicting properties of the rat somatosensory system by sparse coding," Neural Inform. Process. Lett. Rev., vol. 4, pp. 11-18, 2004.

[30] M. Kaneko and T. Tsuji "A whisker tracing sensor with $5 \mu \mathrm{m}$ sensitivity," in Proc. 2000 IEEE/RSJ Int. Conf. Intell. Robots Syst., Takamatsu, Japan, pp. 3907-3912.

[31] O. Bebek and M. C. Cavusoglu, "Whisker sensor design for three dimensional position measurement in robotic assisted beating heart surgery," in Proc. 2007 IEEE Int. Conf. Robot. Autom., Rome, Italy, pp. 225231.

[32] T. Tsujimura and T. Yabuta, "Object detection by tactile sensing method employing force/torque information," IEEE Trans. Robot. Autom., vol. 5, no. 4, pp. 444-450, Aug. 1989.

[33] R. A. Russell, "Using tactile whiskers to measure surface contours," in Proc. 1992 IEEE Int. Conf. Robot. Autom., Nice, France, pp. 12951299.

[34] J. Wilson and Z. Chen, "A whisker probe system for shape perception of solids," ASME J. Dyn. Syst., Meas., Control, vol. 117, pp. 104-108, 1995.

[35] N. Ueno, M. Svinin, and M. Kaneko, "Dynamic contact sensing by flexible beam," IEEE/ASME Trans. Mechatron., vol. 3, no. 4, pp. 254-264, Apr. 1998.

[36] R. Russell and J. Wijaya, "Object location and recognition using whisker sensors," presented at the 2003 Australian Conf. Robot. Autom., Brisbane, Australia.

[37] T. N. Clements and C. D. Rahn, "Three dimensional contact imaging with an actuated whisker," IEEE Trans. Robot., vol. 22, no. 4, pp. 844-848, 2006.

[38] M. Kaneko, N. Kanayama, and T. Tsuji, "Vision-based active sensor using a flexible beam," IEEE/ASME Trans. Mechatron., vol. 6, no. 1, pp. 7-16, Mar. 2001.

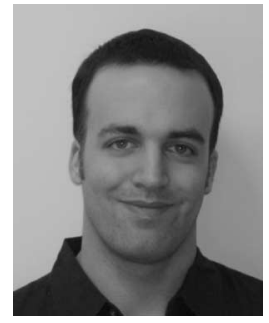

Joseph H. Solomon received the B.S. degree in aeronautical and astronautical engineering from the University of Illinois at Urbana-Champaign, UrbanaChampaign, in 2001, the M.S. degree in mechanical engineering from the University of Illinois at Chicago, Chicago, in 2003, and the Ph.D. degree in mechanical engineering from the Northwestern University, Evanston, IL, in 2008.

$\mathrm{He}$ is currently a Post-Doctoral Scholar with the Mechanical Engineering Department at Northwestern University, Evanston, IL. His research interests include evolutionary robotics, neural networks, biomimetic sensing, and bipedal locomotion.

Dr. Solomon is currently a member of the American Association for the Advancement of Science.

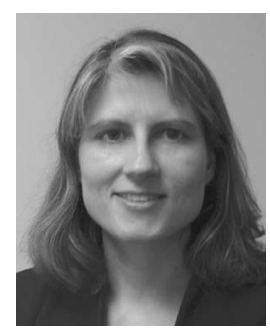

Mitra J. Z. Hartmann received the B.S. degree in applied and engineering physics from Cornell University, Ithaca, NY, and the Ph.D. degree in integrative neuroscience from California Institute of Technology, Pasadena.

During 2000-2003, she was a Postdoctoral Scholar at the Jet Propulsion Laboratory, Pasadena, where she worked in the Bio-Inspired Technology and Systems Group. She is currently with the Departments of Biomedical Engineering and Mechanical Engineering, Northwestern University. She is also a member of the Northwestern University Institute for Neuroscience (NUIN).

Dr. Hartmann is a member of the Scientific Programming Committee for the annual Barrels conference. She is also a member of the editorial board of the Journal of Neurophysiology, and a Review Editor for the Frontiers in Neurorobotics. She is a member of the Biomedical Engineering Society, the American Society of Mechanical Engineers, the Society for Neuroscience, and the Society of Women Engineers. 